\title{
Classification of targets for gamma-ray dark matter searches with speed-dependent annihilation and substructure boost
}

\author{
Thomas Lacroix, ${ }^{a}$ Gaétan Facchinetti, ${ }^{a}$ Judit Pérez-Romero, ${ }^{b}$ Martin Stref, ${ }^{c, *}$ Julien \\ Lavalle, $^{a}$ David Maurin $^{d}$ and Miguel A. Sánchez-Conde ${ }^{b}$ \\ ${ }^{a}$ Laboratoire Univers et Particules de Montpellier (LUPM), Université de Montpellier \& CNRS, Place \\ Eugène Bataillon, 34095 Montpellier Cedex 05, France \\ ${ }^{b}$ Instituto de Física Teórica (IFT) UAM/CSIC, Universidad Autónoma de Madrid, Calle Nicolás Cabrera, \\ 13-15, 28049 Madrid, Spain \\ ${ }^{c}$ Laboratoire d'Annecy-le-Vieux de Physique Théorique (LAPTh), Université Grenoble Alpes, Université \\ Savoie Mont Blanc, CNRS, F-74000 Annecy, France \\ ${ }^{d}$ Laboratoire de Physique Subatomique \& Cosmologie (LPSC), Université Grenoble Alpes, CNRS/IN2P3, \\ 53 avenue des Martyrs, 38026 Grenoble, France
}

E-mail: martin.stref@lapth.cnrs.fr

Indirect searches with gamma rays set strong constraints on the properties of self-annihilating dark matter particle candidates. Observations of dwarf galaxies and galaxy clusters are especially interesting. Here we revisit the calculation of the annihilation flux by considering a velocitydependent cross-section (Sommerfeld enhanced, for both $s$-wave and $p$-wave) and the boost factor due to dark matter substructure. We show that the combination of these two effects can lead to dramatic enhancements of the gamma-ray flux, and possibly modify the hierarchy between the brightest targets in the sky.

$37^{\text {th }}$ International Cosmic Ray Conference (ICRC 2021)

July 12th-23rd, 2021

Online - Berlin, Germany

\footnotetext{
*Presenter
} 


\section{Introduction}

Astrophysical observations are a prime avenue to shed light on the nature of dark matter (DM), whether it is made of particles, macroscopic object or it is due to our lack of understanding of gravity. In the case of particle DM candidates, indirect searches using multiple probes have led to complementary constraints on the underlying properties of the particle candidate. Gamma-ray searches in particular have proven to be a powerful tool to constrain scenarios where the DM can self-annihilate [1].

In the context of gamma-ray searches, most of the effort has been dedicated to the study of the simplest weakly interacting massive particle (WIMP) scenario where the DM particle has a velocity-independent ( $s$-wave) annihilation cross-section set by the relic abundance. However the absence of any confirmed detection has lead to a broadening of search strategies in the recent years. In particular, many theoretical models predict a velocity-dependent annihilation crosssection which leads to a richer phenomenology which we investigate here. For instance, if the DM annihilates through a scalar mediator, annihilation proceeds through a $p$-wave process which leads to a strong velocity dependence $\sigma v_{\text {rel }} \propto v_{\text {rel }}^{2}$ (see e.g. [2]). Another interesting configuration arises if annihilation is mediated by a particle lighter than the DM. In the presence of an attractive potential, this leads to the Sommerfeld effect which can greatly enhance the cross section and create a specific velocity dependence. The phenomenology of DM can also be complicated on the astrophysics side. Cold particle candidates are expected to collapse on very small scales which leads to a vast population of substructures within galactic DM halos [3]. These small subhalos have long been known to impact predictions for velocity-independent annihilation, however less attention has been given to their interplay with velocity-dependent annihilation.

This study is dedicated to the prediction of the astrophysical factor governing the DM-induced gamma-ray flux, including velocity dependence and subhalos. The different theoretical ingredients needed for the calculation are presented in Sec. 2. Several targets of interest covering different mass range (dwarf spheroidal galaxies and galaxy clusters) are considered and presented in Sec. 3. We investigate how the particle models and the modeling of subhalos impact the ranking of these targets in Sec. 4. This proceedings is based on Lacroix et al. 2021 (to be published).

\section{Theoretical ingredients}

\subsection{Velocity-dependent annihilation}

We consider the phenomenological scenario in which DM particles self-interact through the exchange of a (light) mediator $\phi$ of mass $m_{\phi}$ with coupling $g_{X}=\sqrt{4 \pi \alpha_{X}}$, where $\alpha_{X}$ plays the role of dark fine structure constant (we fix $\alpha_{X}=10^{-2}$ in the following). This long-range interaction modifies the $s$-wave annihilation cross-section in the following way

$$
\sigma v_{\text {rel }}=\left(\sigma v_{\text {rel }}\right)_{0} \times S
$$

where $S$ can be evaluated by solving Schrödinger's equation for an attractive Yukawa potential. The Sommerfeld factor $S$ depends on the velocity through a parameter $\epsilon_{v}=v /\left(c \alpha_{X}\right)$, and the masses and coupling through a parameter $\epsilon_{\phi}=m_{\phi} /\left(\alpha_{X} m_{\chi}\right)$. Its shape depends on the partial wave i.e. 
there is a factor $S_{s}$ for $s$-wave and a different factor $S_{p}$ for $p$-wave. Although these factors have a complicated expression, different asymptotic regimes can be identified

- if $\epsilon_{v} \gg 1$, there is no enhancement $S_{s} \simeq S_{p} \simeq 1$

- if $\epsilon_{\phi} \gg 1$, there is again no enhancement

- if $\epsilon_{\phi} \ll \epsilon_{v} \ll 1$, this corresponds to the Coulomb regime, $S_{s} \propto 1 / v$ and $S_{p} \propto 1 / v^{3}$, and there is no dependence in $\epsilon_{\phi}$

- if $\epsilon_{v} \ll \epsilon_{\phi} \ll 1$, the Sommerfeld factor becomes almost independent of the velocity, except on a series of resonances at $\epsilon_{\phi}=6 /\left(\pi^{2} n^{2}\right)$ with $n$ an integer where $S_{s}$ and $S_{p}$ are proportional to $1 / v^{2}$

In practice, instead of numerically solving Schrödinger's equation for a Yukawa potential, we use the analytic solution available for a Hulthén potential which is very similar [4].

\subsection{Dark matter subhalos and phase-space modeling}

To compute the enhancement of the annihilation rate induced by substructures, we use the analytical model of ref [5]. This model allows to compute the parameter-space density function of subhalos (which depends on the concentration, mass and position) inside any given DM halo. It relies on theoretical inputs from cosmology (Seth-Tormen halo mass function, concentration distribution) and gravitational dynamics (tidal effects). The minimal subhalo mass, which is in principle related to the kinetic decoupling of the DM particle [6], is a free parameter of the model and is set to $10^{-6} \mathrm{M}_{\odot}$.

Let $f\left(\vec{r}, \vec{v}_{\text {rel }}\right)$ be the relative phase-space distribution function (pdf) of DM particles inside a subhalo, then a Sommerfeld-enhanced $s$-wave cross-section leads to a luminosity

$$
L_{S} \propto \int \rho^{2}(\vec{r})\left[\int f\left(\vec{r}, \vec{v}_{\text {rel }}\right) S_{S}\left(v_{\text {rel }}\right) \mathrm{d}^{3} \vec{v}_{\text {rel }}\right] \mathrm{d}^{3} \vec{r} .
$$

In practice, rather than computing the phase-space pdf for each subhalo, we make the following approximations

$$
\int f\left(\vec{r}, \vec{v}_{\text {rel }}\right) S_{S}\left(v_{\text {rel }}\right) \mathrm{d}^{3} \vec{v}_{\text {rel }} \simeq S_{S}\left[\left\langle v_{\text {rel }}^{-2}\right\rangle^{-1 / 2}\right] \text { with }\left\langle v_{\text {rel }}^{-2}\right\rangle=\int f\left(\vec{r}, \vec{v}_{\text {rel }}\right) v_{\text {rel }}^{-2} \mathrm{~d}^{3} \vec{v}_{\text {rel }},
$$

where the relative velocity moment is computed using Eddington's inversion method [7]. Similar expressions and approximations are established for the Sommerfeld-enhanced $p$-wave annihilation case. The luminosity of the full object is obtained by integrating $L_{S}$ over the subhalo parameter-space density function and adding the contribution of the smooth DM component.

\section{Targets}

\subsection{Dwarf spheroidal galaxies}

Due to their close proximity (a few tens of kpc), their high DM density and low astrophysical background, dwarf spheroidal galaxies (dSphs) in the Milky Way are among the most interesting 
targets for DM indirect searches. DSphs are separated in two categories, the classical and the ultra-faints. In this study, we pick three classical dSphs (Draco, Ursa Minor and Sculptor) and two ultra-faint dSphs (Ursa Major II and Coma Berenices) which were found to be among the best targets for DM annihilation [8]. In the latter study, the DM profile parameters were reconstructed from a Markov Chain Monte Carlo (MCMC) engine coupled to the CLUMPY $\operatorname{code}^{1}[9,10]$. The DM density profile in the $\mathrm{dSphs}$ is modeled using the Einasto profile.

\subsection{Galaxy clusters}

Galaxy clusters are the largest gravitationally-bound objects in the Universe. Their masses are between $10^{14}-10^{15} \mathrm{M}_{\odot}$ and up to $80 \%$ of this mass is DM. The rest is baryonic matter in the form of galaxies, gas and dust. Although some gamma-ray emission is expected due to cosmic-ray activity, clusters still constitute excellent targets for indirect searches. DM subhalos play an important in these objects since the boost to the annihilation signal is expected to be much larger than in dSphs. We rely on ref [11] and pick three galaxy clusters (Coma, Fornax and Perseus) for the present study. The DM profile of the clusters is modeled by the NFW profile. Galaxy cluster mass measurements are subject to large uncertainties, hence we rely on two different estimates for each cluster in our analysis. One estimate stems from X-ray measurements ("hydro" mass) while the other is based on Sunyaev-Zeldovich [12].

\section{Results}

Elements related to astrophysics in the expression of the DM-induced gamma-ray flux are contained within the so-called $J$-factor along a given line of sight (l.o.s)

$$
J=\int_{\Delta \Omega} \mathrm{d} \Omega \int_{\text {l.o.s. }} \mathrm{d} s \rho_{\text {eff }}^{2}(s),
$$

where $\rho_{\text {eff }}$ is an effective DM density. If the annihilation is $s$-wave and subhalos are ignored, $\rho_{\text {eff }}$ is equal to the true DM density, otherwise it has to be modified as discussed in Sec. 2. We show the $J$-factor for the Sommerfeld case as a function of $\epsilon_{\phi}$ in Fig. 1. We recall that when $\epsilon_{\phi} \gg 1$, which corresponds to rightmost part of each panel, there is no Sommerfeld enhancement. Let us focus on $\mathrm{dSphs}$ first which are shown in the upper panel. The solid curves show the result including subhalos while the dashed curves show the result assuming all the DM is smooth. When Sommerfeld effect is turned off, subhalos only provide a very small boost, of order a few tens of percent, and the dashed and solid curve are very close. In a dwarf galaxy without clumps, we typically have $\epsilon_{v} \simeq 10^{-2}$ so when $\epsilon_{\phi}>10^{-2}$ we are in the resonant regime and when $\epsilon_{\phi}<10^{-2}$ we are in the Coulomb regime. This can be seen on the dashed curves. However, the presence of subhalos implies much lower values of $\epsilon_{v}$ can be reached because the velocity dispersion is very low in these objects. This means that the resonant regime extends to much lower values of $\epsilon_{\phi}$, hence the $J$-factor can reached much higher values compared to the smooth case. The enhancement can be very large even in-between resonances because $S \propto 1 / \epsilon_{\phi}$ in this case while $S$ does not depend on $\epsilon_{\phi}$ in the Coulomb regime. The phenomenology is essentially the same in the $p$-wave case, although the amplitude is strongly suppressed compared to $s$-wave and the shape is slightly different.

\footnotetext{
1https://clumpy.gitlab.io/CLUMPY/
} 
For galaxy clusters, which are shown in the bottom panel of Fig. 1, the trend is the same but the effect is even more dramatic because the subhalo boost factor is much larger than in dSphs for $s$-wave annihilation. Even without Sommerfeld enhancement, the boost can be as high as 30 in the case of the Fornax cluster. The boost takes much larger values at low $\epsilon_{\phi}$, reaching several orders of magnitude. Similar observations can be made in the $p$-wave case, with the amplitude being again suppressed compared to $s$-wave.
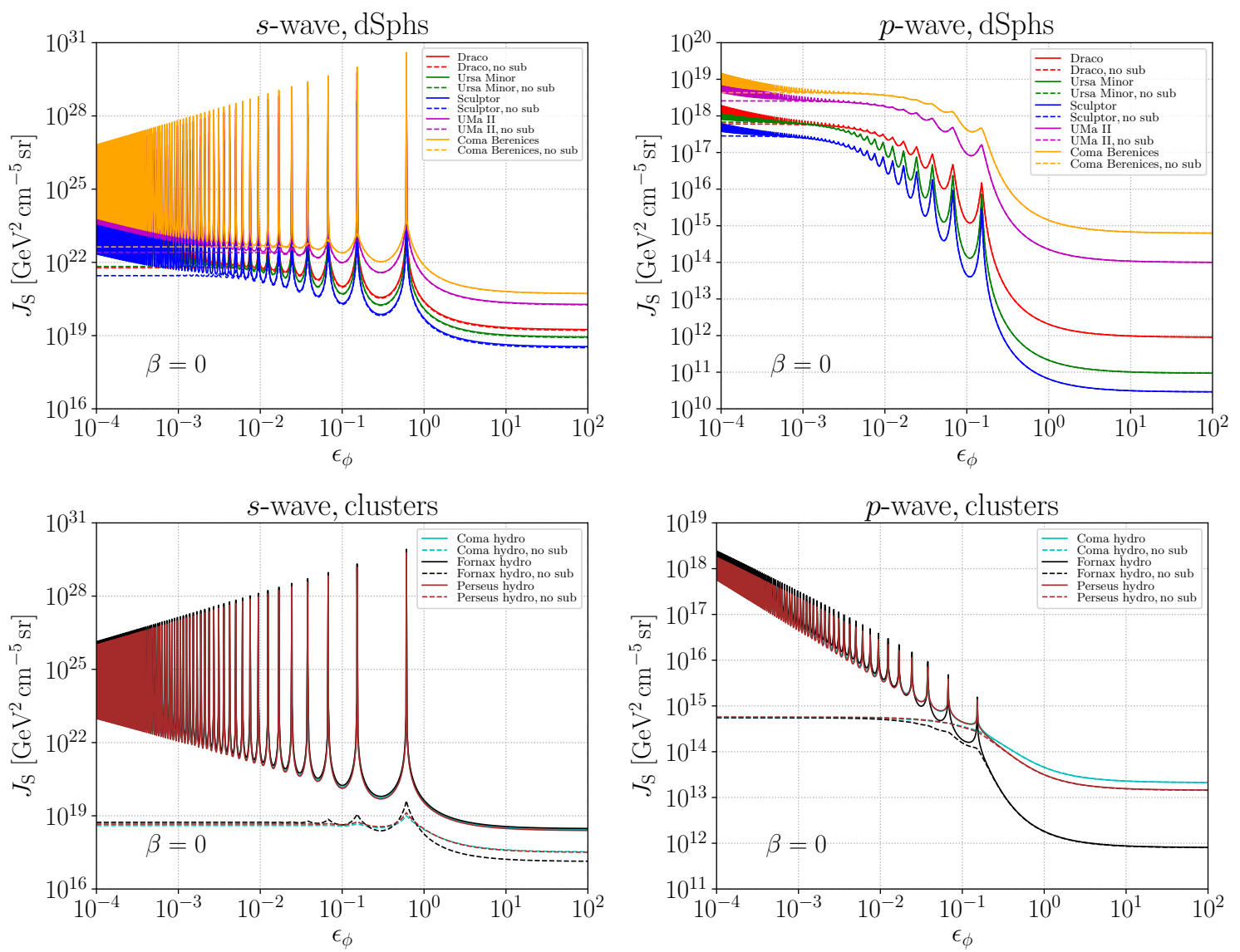

Figure 1: $J$-factor as a function of $\epsilon_{\phi}$, with and without subhalos. The top panels show the results for dSphs and the bottom panels show the results for galaxy cluster. The left panels show the $s$-wave case and the right panels show the $p$-wave.

In Fig. 2, the $J$-factor of an object is shown for each of the source category we consider : Fornax for the galaxy clusters, Draco for the classical dSphs, Ursa Major II for the ultra-faints dSphs, and IC10 for the dIrrs. Looking at $s$-wave first, without subhalos, we see that for $\epsilon_{\phi} \gg 1$ we have $J_{\text {Fornax }}<J_{\text {IC10 }}<J_{\text {UMaII }}<J_{\text {Draco. }}$. If substructures are included, $J_{\text {Fornax }}$ is significantly boosted and gets very close to $J_{\mathrm{IC} 10}$. Going down to lower values of $\epsilon_{\phi}$, the $J$-factors of the different targets get closer and closer. The same behavior is observed for $p$-wave with a different hierarchy : here we have $J_{\mathrm{IC} 10}<J_{\text {Fornax }}<J_{\text {Draco }}<J_{\text {UMaII }}$. As $\epsilon_{\phi}$ gets lower, the annihilation in the most massive objects (Fornax and IC10) get significantly boosted and catches up with the annihilation in the lightest ones.

The interplay between the Sommerfeld effect and subhalos not only impacts the hierarchy 

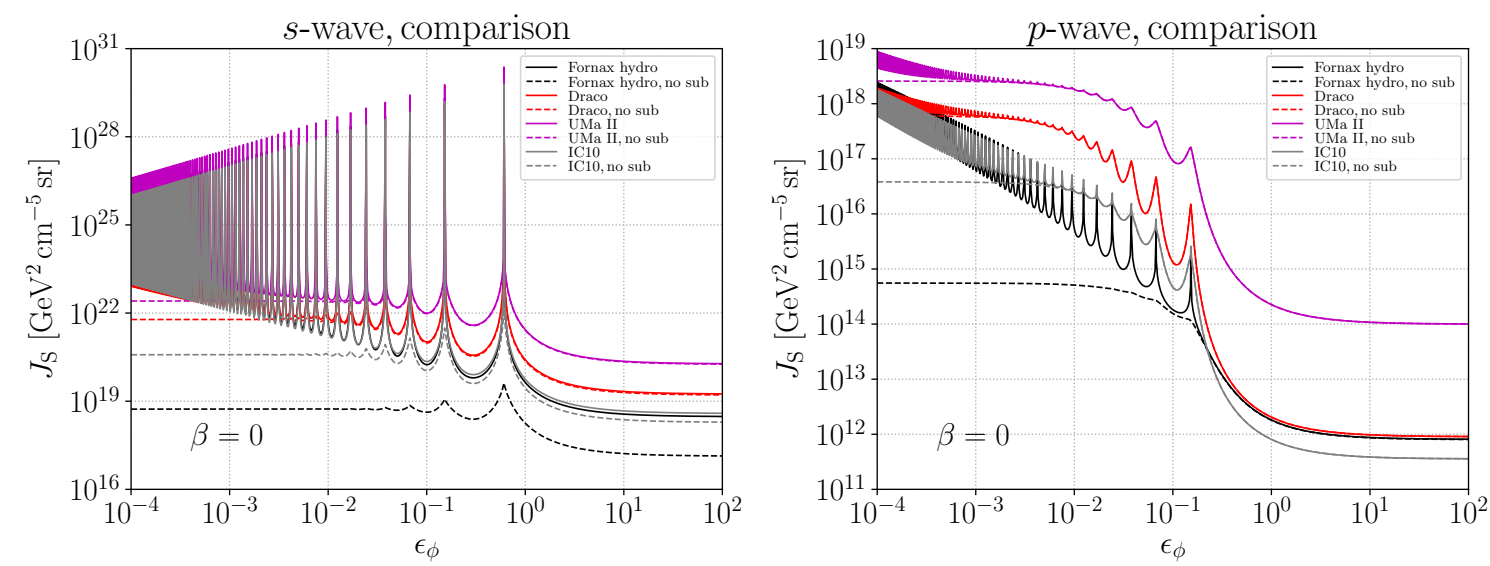

Figure 2: $J$-factors comparison for all targets. The left panel shows the $s$-wave case and the right panel shows the $p$-wave case.

between targets of interest but can also impact their detectability above the Milky Way (MW) halo foreground annihilation. This annihilation is also impacted by Sommerfeld enhancement and subhalos. We have computed this contribution as a function of the angle $\psi_{0}$ between the line of sight and the Galactic plane. The result is shown as the blue solid line in Fig. 3 along with the $J$ factor of each object in the study. The integration angle is $0.5^{\circ}$. The case $\epsilon_{\phi} \gg 1$ (i.e. without Sommerfeld) is shown in the left panel while the right panel shows the results for $\epsilon_{\phi} \simeq 10^{-4}$ (off-resonances). We see that some targets can be above or below the MW foreground depending on whether the Sommerfeld effect in plugged-in or not. For instance, Fornax is slightly below the blue curve in the left panel, but slightly above the right panel.
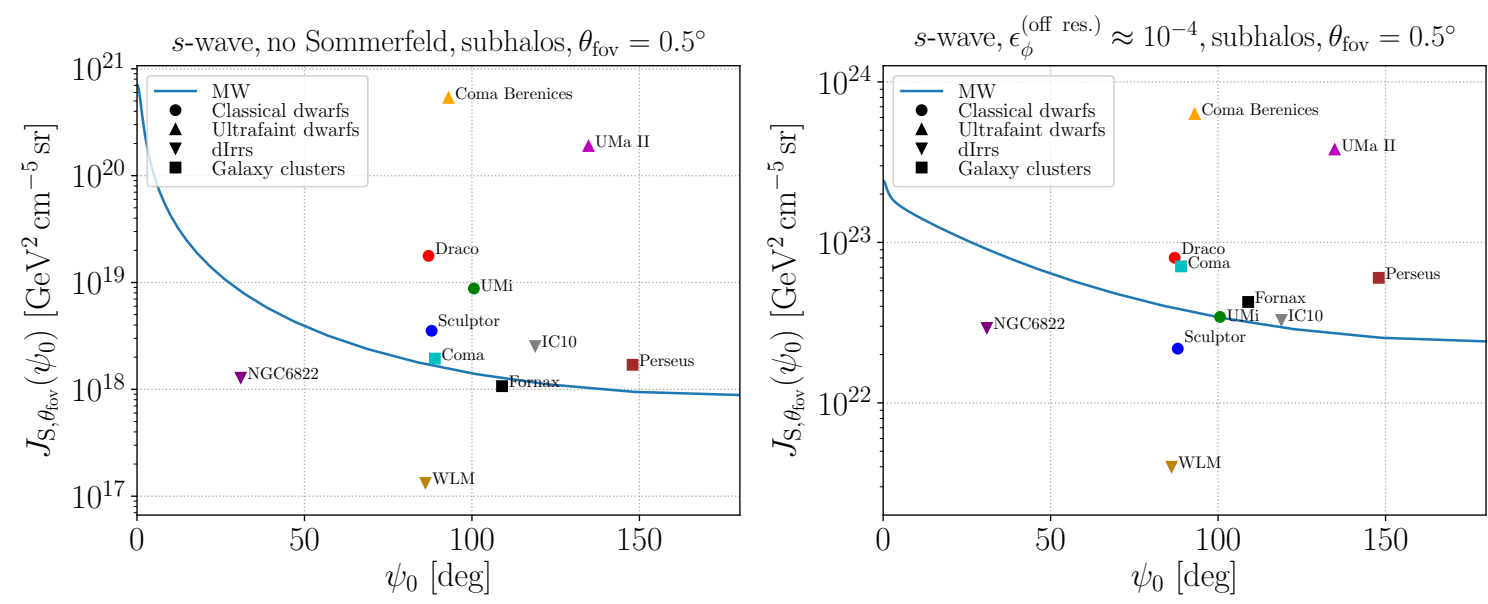

Figure 3: Comparison between $J$-factors of the various targets in this work and the expected foreground from DM annihilation in the Milky Way halo. 


\section{Conclusion}

In this study, we have achieved a consistent calculation of the astrophysical factor for gammaray searches including a Sommerfeld-enhanced annihilation cross-section and the effect of DM subhalos. We have shown that these two effects combine can lead to a dramatic increase of the expected gamma-ray flux from targets of interest such as dwarf galaxies and galaxy clusters. We have explicitly computed the $J$-factors for some of these objects and shown that the hierarchy in terms of luminosity can also be impacted. Finally, we have compared these luminosities to the foreground annihilation from the MW DM halo and found that some targets can be either below or above that foreground depending on the value of the particle physics parameters. In future works, we will discuss how these results impact the constraints on the DM microscopic properties.

\section{Acknowledgments}

TL has received funding from the European Union's Horizon 2020 research and innovation program under the Marie Skłodowska-Curie grant agreement No. 713366. The work of TL, MASC and JPR is supported by the Spanish Agencia Estatal de Investigación through the grants PGC2018095161-B-I00, IFT Centro de Excelencia Severo Ochoa SEV-2016-0597, and Red Consolider MultiDark FPA2017-90566-REDC. GF and JL are partly supported by the ANR projectANR-18CE31-0006, the OCEVU Labex (ANR-11-LABX-0060), the national CNRS-INSU programs PNHE and PNCG, and European Union's Horizon 2020 research and innovation program under the Marie Sklodowska-Curie grant agreements No 690575 and No 674896 - inaddition to recurrent funding by CNRS-IN2P3 and the University of Montpellier. MS is supported by the University Grenobles Alpes. DM is supported by the CNRS.

\section{References}

[1] S. Hoof, A. Geringer-Sameth and R. Trotta, A Global Analysis of Dark Matter Signals from 27 Dwarf Spheroidal Galaxies using 11 Years of Fermi-LAT Observations, JCAP 02 (2020) 012, [1812.06986].

[2] M. Boudaud, T. Lacroix, M. Stref and J. Lavalle, Robust cosmic-ray constraints on p-wave annihilating MeV dark matter, Phys. Rev. D 99 (2019) 061302, [1810 . 01680].

[3] V. Springel, J. Wang, M. Vogelsberger, A. Ludlow, A. Jenkins, A. Helmi et al., The Aquarius Project: the subhalos of galactic halos, Mon. Not. Roy. Astron. Soc. 391 (2008) 1685-1711, [0809.0898].

[4] S. Cassel, Sommerfeld factor for arbitrary partial wave processes, J. Phys. G 37 (2010) 105009, [0903.5307].

[5] M. Stref and J. Lavalle, Modeling dark matter subhalos in a constrained galaxy: Global mass and boosted annihilation profiles, Phys. Rev. D 95 (2017) 063003, [1610.02233].

[6] A. M. Green, S. Hofmann and D. J. Schwarz, The First wimpy halos, JCAP 0508 (2005) 003, [astro-ph/0503387]. 
[7] T. Lacroix, M. Stref and J. Lavalle, Anatomy of eddington-like inversion methods in the context of dark matter searches, Journal of Cosmology and Astroparticle Physics 2018 (Sep, 2018) 040-040.

[8] V. Bonnivard et al., Dark matter annihilation and decay in dwarf spheroidal galaxies: The classical and ultrafaint dSphs, Mon. Not. Roy. Astron. Soc. 453 (2015) 849-867, [1504.02048].

[9] V. Bonnivard, M. Hütten, E. Nezri, A. Charbonnier, C. Combet and D. Maurin, CLUMPY : Jeans analysis, $\gamma$-ray and $v$ fluxes from dark matter (sub-)structures, Comput. Phys. Commun. 200 (2016) 336-349, [1506. 07628].

[10] M. Hütten, C. Combet and D. Maurin, CLUMPY v3: $\gamma$-ray and $v$ signals from dark matter at all scales, Comput. Phys. Commun. 235 (2019) 336-345, [1806 . 08639].

[11] M. A. Sánchez-Conde, M. Cannoni, F. Zandanel, M. E. Gómez and F. Prada, Dark matter searches with cherenkov telescopes: nearby dwarf galaxies or local galaxy clusters?, Journal of Cosmology and Astroparticle Physics 2011 (Dec, 2011) 011-011.

[12] G. Schellenberger and T. H. Reiprich, Hicosmo - cosmology with a complete sample of galaxy clusters - $i$. data analysis, sample selection and luminosity-mass scaling relation, Monthly Notices of the Royal Astronomical Society 469 (Apr, 2017) 3738-3761. 